\title{
Génétique des populations de chats domestiques dans un district rural du Cher (France)
}

\author{
Ph. DREUX \\ Laboratoire de Zoologie, Ecole Normale Supérieure \\ 46, rue d'Ulm, F-75230 Paris Cedex 05
}

\begin{abstract}
Résumé
Un échantillonnage de 400 chats a été examiné dans des fermes et de petits villages d'un district rural du Cher. La population n'a pas montré d'écart significatif à la panmixie. Les fréquences des gènes mutants ont été estimées et les classes de panachure notées. Une comparaison avec quelques autres populations déjà étudiées en France est en faveur de l'hypothèse que les gènes $t^{b}$ et $O$ ont des valeurs sélectives différentes en milieu rural et en milieu urbain.
\end{abstract}

\section{Introduction}

Parmi les nombreux travaux déjà publiés qui ont été faits pour estimer la fréquence des gènes mutants dans des populations de chats domestiques, la plupart concernaient des villes. Il est en effet beaucoup plus facile et plus rapide d'y recueillir les données nécessaires car les populations de chats sont denses et les individus plus faciles à examiner. Les prospections chez des vétérinaires, ou dans des refuges pour animaux abandonnés permettent souvent d'en voir beaucoup en peu de temps, et dans de bonnes conditions d'observation.

En milieu rural, les investigations sont beaucoup plus longues et nécessitent des déplacements qui diminuent encore le rendement d'un tel travail. Il est donc normal que jusqu'ici peu de sondages aient été entrepris à la campagne ; le seul qui ait porté sur un effectif important, comparable à l'étude soignée d'une population de grande ville, a été fait en France, dans un district rural de Mayenne (Dreux, 1968), par suite de circonstances favorables. D'autres études en milieu rural n'ont porté que sur des effectifs inférieurs à la centaine (TodD, ClARK \& Dreux, 1974) alors que celui du sondage précité dépasse 600 .

Cependant, l'étude de populations rurales est très importante. Une revue générale récente des fréquences géniques dans les populations d'Europe (ToDD, 1977) a montré pour plusieurs locus des clines très nets dans la fréquence respective des allèles. Mais l'existence de ces clines repose à peu près uniquement sur des sondages réalisés en milieu urbain, et il a souvent été affirmé (ToDD, 1969) que l'environnement d'une 
ville exercerait sur certains locus une pression de sélection assez forte, en principe en faveur des allèles amenant un pelage plus foncé. Pour vérifier ou infirmer cette hypothèse, des recherches plus sérieuses sont nécessaires, et c'est la raison du présent travail.

\section{Méthodes}

Les investigations ont été faites de 1973 à 1981 dans l'est du département du Cher, tout près du centre de la France. Le territoire prospecté représente grossièrement un rectangle bordant la Loire à l'ouest sur $20 \mathrm{~km}$ de largeur et s'étendant environ sur $50 \mathrm{~km}$ entre les localités de Sancerre au nord et Sancoins au sud. Tous les chats notés ont été vus dans des fermes isolées, des hameaux et des villages de moins de 3000 habitants. Le sexe des animaux était noté chaque fois qu'il a pu être reconnu de façon certaine.

Le polymorphisme de ces chats a été étudié par rapport aux facteurs génétiques connus, qui portent surtout sur la couleur et la longueur du pelage (RoBinson, 1959 ; SEARLE, 1968 ; Committee on Nomenclature, 1968) :

- $a$, non agouti, épistatique sur $t$,

- $d$, dilution maltaise,

- $l$, persan : poils longs,

- $O$, orange, lié au sexe, épistatique sur $a$; les femelles hétérozygotes $O /+$ ont le phénotype écaille-de-tortue, où des plages oranges se juxtaposent avec des plages normalement colorées,

- $S$, panachure blanche irrégulière, semi-dominant à expressivité variable,

- $t^{b}$, tigré large, allèle récessif de $t^{+}$, tigré ordinaire rayé,

- $W$, blanc dominant, épistatique sur toutes les autres couleurs.

L'allèle $T^{a}$ (tigré type Abyssinie) au locus $t$ n'a pas été rencontré dans cet échantillonnage. Aucun chat ne portait la dilution siamoise $c^{s}$, allèle de la série d'albinisme, récessif par rapport à l'allèle normal $C$. On a observé 8 individus «chinchilla» (sur phénotype tigré) et 2 «argentés 》 (sur phénotype non-agouti) ; le déterminisme de ce facteur considéré classiquement comme allèle du locus $C$ étant douteux, il n'a pas été étudié au point de vue de la fréquence génique. Sur les 17 chats rencontrés porteurs du gène blanc dominant $W, 12$ seulement ont pu être approchés d'assez près pour pouvoir déterminer la couleur des yeux; un seul avait les yeux bleus, les autres les yeux jaunes; aucun cas de dissymétrie n'a été observé.

Au point de vue des anomalies squelettiques, il est à noter qu'aucun chat à queue tordue (Kinky-tail) n'a été observé avec certitude; un individu avait une queue très courte, environ moitié de la normale, mais cela pouvait très bien être dû à un accident. Ceci est à rapprocher de l'absence de siamois dans l'échantillonnage, ceux-ci portant souvent ce gène et le répandant dans les populations lorsqu'ils participent à la reproduction. D'autre part, aucun individu polydactyle ou syndactyle n'a été repéré, mais, beaucoup d'individus n'ayant pas été examinés de près, cette conclusion n'a rien de certain. 


\section{Résultats}

\section{1. - Généralités}

Les investigations ont été arrêtées lorsque le nombre fixé à l'avance de 400 individus a été atteint. Sur ceux-ci, 77 étaient des jeunes n'ayant pas atteint leur taille définitive et la maturité sexuelle. Ce nombre était trop faible pour faire une comparaison jeunes-adultes dans le but de voir si une sélection humaine jouait et d'ailleurs les résultats en auraient été difficiles à interpréter, car si certaines portées sont en partie sacrifiées par le propriétaire de leur mère, beaucoup échappaient certainement à toute intervention.

Aucune femelle ne pouvait être déterminée clairement comme stérilisée et il n'a été vu que 2 mâles dont la castration était évidente. Il semble bien que la grande majorité des individus participent effectivement à la reproduction au sein de la population.

Sur les 400 chats rencontrés, 124 ont été clairement déterminés comme mâles, 171 comme femelles et le sexe de 105 n'a pu être reconnu. L'excès de femelles reconnues tient certainement pour une grande part aux 49 phénotypes écaille-de-tortue, automatiquement classées comme telles. Par suite, on admettra raisonnablement, semble-t-il, dans ce qui suit, que la proportion des mâles dans la population n'est pas statistiquement significativement différente de 50 p. 100.

\section{2. - Test de la panmixie sur le gène orange $\mathrm{O}$}

Tous les individus examinés peuvent être testés sur les allèles de ce gène, à l'exception des 17 individus ( 5 mâles, 8 femelles, 4 de sexe inconnu) portant le gène $W$, blanc dominant, épistatique sur tous les gènes de coloration, en particulier $O$; il en restait donc 383 classifiables pour le gène orange. Les 49 femelles écailles-de-tortue étaient facilement reconnaissables ; un gros effort d'approche a été fait pour déterminer le sexe des chats de phénotype orange ; sur 20,18 étaient mâles, 2 seulement femelles.

En admettant que les 383 individus classifiables se répartissaient également entre les deux sexes (191 mâles et 192 femelles), ils possédaient 575 chromosomes X dont 71 porteurs du gène orange et 504 du gène sauvage; d'où la fréquence du gène orange, 0,1235 , et de son allège sauvage, 0,8765 . On a calculé sur cette base les fréquences théoriques des différents phénotypes en admettant que la population était panmictique et que le gène $O$ s'y conformait donc à la loi de Hardy-Weinberg. Elles sont présentées dans le tableau 1, en comparaison avec les fréquences observées; celles-ci montrent par rapport aux prévisions un déficit de mâles orange et un excès de femelles écaille-de-tortue. Cependant, le $\chi^{2}$ total n'est que de 3,42 pour 2 degrés de liberté, et, même au seuil de probabilité de 10 p. 100 , on n'a pas de raison statistiquement valable de douter de la panmixie de la population pour le gène $O$. 


\section{TABleau 1}

Distributions observées et calculées (entre parenthèses) des phénotypes correspondant au gène orange dans la population de chats étudiée

Observed and expected (in brackets) distribution of phenotypes corresponding to the orange gene in the cat population studied

\begin{tabular}{|c|c|c|c|}
\hline Génotypes & $O /$ ou $O / O$ & $\mathrm{O} /+$ & $+/-$ ou $+/+$ \\
\hline Mâles (191) & $18(23,59)$ & 0 & $173(167,41)$ \\
\hline Femelles (192) & $2 \quad(2,93)$ & $49(41,57)$ & $141(147,50)$ \\
\hline
\end{tabular}

\section{3. - Fréquence des gènes}

En supposant que la population étudiée était panmictique également pour les autres gènes, on a calculé la fréquence de leurs allèles mutants. Ces fréquences sont données dans le tableau 2 où figure aussi celle de $O$. Les effectifs phénotypiques qui y sont mentionnés sont ceux de l'homozygote pour les gènes récessifs (exemple : $a a$ ), ceux de l'homozygote et de l'hétérozygote pour les gènes dominants (exemple : $[W]$ ). L'intervalle de confiance avec une probabilité de 95 p. 100 a été donné dans la dernière colonne, la distribution pour des gènes rares n'étant pas symétrique autour de la moyenne.

\section{TABleau 2}

Fréquence des gènes dans la population de chats étudiée

Gene frequencies in the cat population studied

\begin{tabular}{|c|c|c|c|c|c|}
\hline Allèles & $\begin{array}{l}\text { Nombres } \\
\text { classifiables }\end{array}$ & $\begin{array}{c}\text { Effectifs } \\
\text { phénotypiques }\end{array}$ & $\begin{array}{c}\text { Fréquences } \\
\text { phénotypiques } \\
(\text { en } \%)\end{array}$ & $\begin{array}{l}\text { Fréquences } \\
\text { géniques }\end{array}$ & $\begin{array}{c}\text { Intervalle } \\
\text { de confiance } \\
\text { à } 95 \%\end{array}$ \\
\hline$a$ & 363 & 176 & 0,43 & 0,70 & $0,66-0,73$ \\
\hline$d \ldots \ldots$ & 383 & 52 & 0,14 & 0,37 & $0,32-0,42$ \\
\hline$l \ldots \ldots$ & 400 & 16 & 0,04 & 0,20 & $0,16-0,25$ \\
\hline$o \ldots \ldots$ & 383 & 一 & - & 0,12 & $0,10-0,15$ \\
\hline$s \ldots \ldots$ & 383 & 168 & 0,44 & 0,25 & $0,22-0,29$ \\
\hline$t^{+}$ & 193 & 120 & 0,62 & 0,385 & $0,33-0,44$ \\
\hline$t^{b^{e}} \ldots$ & 193 & 73 & 0,38 & 0,615 & $0,56-0,67$ \\
\hline$W \quad \ldots$ & 400 & 17 & 0,042 & 0,021 & $0,013-0,034$ \\
\hline
\end{tabular}




\section{4. - Panachure}

Tous les chats qui ne présentaient pas le phénotype blanc dominant $W$ ont été classés au moyen de l'échelle de panachure déjà définie (Dreux, 1967) dans des catégories de 0 à 0,9 , les nombres exprimant approximativement la fraction du corps de couleur blanche (tabl. 3). On a aussi classé à tout hasard dans une catégorie spéciale les chats présentant sous la gorge ou le ventre (ou les deux) une petite touffe de poils blancs, déjà notée panachure $\varepsilon$; elle était sûrement présente chez au moins 30 chats qui ont été classés par la suite avec ceux qui ne présentaient pas la panachure irrégulière. Le déterminisme de cette panachure discrète est encore mal connu. Au contraire, on sait que la panachure irrégulière est due à un gène semidominant $S$, les homozygotes $S S$ étant en moyenne plus panachés de blanc que les hétérozygotes $S$ s.

\section{TABleau 3}

Classe de répartition de la panachure dans la population

Classes for white-spotting in the cat population studied

\begin{tabular}{r|c|c|c|c|c|c|c|c|c|c|c}
\hline \hline $\begin{array}{c}\text { Classes de panachu- } \\
\text { re e.......... }\end{array}$ & 0 & $\varepsilon$ & 0,1 & 0,2 & 0,3 & 0,4 & 0,5 & 0,6 & 0,7 & 0,8 & 0,9 \\
\hline Effectifs ........ & 185 & 30 & 23 & 22 & 34 & 24 & 12 & 8 & 16 & 17 & 12 \\
\hline
\end{tabular}

La proportion de chats sans panachure irrégulière, donc de génotype ss, permet de calculer la fréquence du gène $S$ dans la population, en admettant que s'applique la loi de Hardy-Weinberg. L'allèle $s$ non panaché est homozygote chez 215 individus sur les 383 classables, il a donc une fréquence de 0,75 et celle de l'allèle $S$ est par suite de 0,25 . L'intervalle de confiance a été calculé comme pour les gènes précédents, auxquels $S$ a été réuni dans le tableau.

\section{Discussion et conclusion}

L'étude de cette population rurale de chats apporte une nouvelle preuve du fait que les fréquences des gènes sont proches de l'équilibre panmictique, au moins pour le locus $O$, dans une grande partie de l'Europe occidentale. En France, ces fréquences ne montrent que des variations faibles, probablement graduelles, et qui dessinent des clines assez réguliers (TodD, 1977).

Les fréquences géniques de cette population de chats du Cher s'inscrivent assez bien dans la moyenne de celles déjà trouvées en France. La comparaison est 
surtout intéressante avec la population rurale déjà étudiée en Mayenne (Dreux, 1968) et les deux populations urbaines étudiées les plus proches, Paris (Dreux, 1967) et Tours (DreuX, 1979) (tabl. 4).

\section{TABleau 4}

Fréquence des gènes dans quelques populations de chats en France

Gene frequencies in some cat population in France

\begin{tabular}{|c|c|c|c|c|c|}
\hline Gènes & $\begin{array}{l}\text { Cher } \\
\text { (rural) }\end{array}$ & $\begin{array}{l}\text { Mayenne } \\
\text { (rural) }\end{array}$ & $\begin{array}{c}\text { Paris } \\
\text { (urbain) }\end{array}$ & $\begin{array}{l}\text { Tours } \\
\text { (urbain) }\end{array}$ & $\begin{array}{c}\text { Marseille } \\
\text { (urbain) }\end{array}$ \\
\hline$a$ & 0,70 & 0,64 & 0,71 & 0,59 & 0,72 \\
\hline$\ldots$ & 0,37 & 0,29 & 0,33 & 0,34 & 0,34 \\
\hline$\ldots$ & 0,20 & 0,17 & 0,24 & 0.27 & 0,27 \\
\hline$\ldots \ldots$ & 0,12 & 0,15 & 0,06 & 0,06 & 0,08 \\
\hline$\ldots$ & 0,25 & 0,39 & 0,24 & 0,22 & 0,29 \\
\hline$t^{b}$ & 0,61 & 0,61 & 0,78 & 0,71 & 0,68 \\
\hline$W$ & 0,02 & 0,01 & 0,01 & 0,02 & 0,003 \\
\hline
\end{tabular}

Pour la plupart des locus, les fréquences ne sont pas différentes de façon statistiquement significatives et en particulier Paris et le district rural du Cher sont très similaires. Deux particularités apparaissent cependant et appellent des commentaires.

Dans les deux populations rurales du Cher et de Mayenne, la fréquence de $t^{b}$ est de 0,61 , très basse vis-à-vis de celles de Paris et de Tours, et même de Marseille située beaucoup plus au sud (Dreux, 1975). Le Cher est à peu près sur la ligne approximativement nord-sud joignant Paris à Marseille et ne s'inscrit donc pas bien dans le cline dessiné par ToDD régulièrement décroissant autour d'un maximum centré sur le sud de l'Angleterre. Ce serait un argument en faveur de l'hypothèse attribuant une valeur sélective plus grande à l'allèle $t^{b}$ en milieu urbain qu'en milieu rural.

L'allèle $O$ a dans le Cher une fréquence élevée - quoique moins qu'en Mayenne s'opposant à celles, très basses, de Paris et de Tours, et même de Marseille. La remarque inverse est donc à faire : bien que le Cher soit proche du couloir ParisMarseille où $O$ est supposé avoir une fréquence particulièrement basse, il ne semble en être influencé que très partiellement, et ceci pourrait s'interpréter par un désavantage moins marqué de l'allèle $O$ en milieu rural, même dans cette zone particulière.

Rien au contraire ne semble étayer l'hypothèse d'un avantage particulier de l'allèle $a$ en milieu urbain, autrefois postulé (ToDD, 1969; ToDD, FAGEN \& FAGEN, 1975 ; ClARK, 1975, etc.) : ses fréquences sont virtuellement identiques dans le Cher et à Paris et sa fréquence la plus basse en France a été rencontrée dans la ville de Tours. 
$\mathrm{Si}$ des clines géographiques à grande échelle pour certains allèles semblent bien exister dans les populations de chats en Europe occidentale, il est fort possible donc que ces clines soient localement modifiés par une différence de valeur sélective pour $O$ et $t^{b}$, entre le milieu rural et les particularités de l'environnement urbain.

Reçu pour publication en octobre 1981.

\section{Summary \\ Gene frequencies in the cat population of a rural district of Cher (France)}

A 400 cats sampling was made in a rural district : lonely farms and small villages. The population was found to be randomly mated. Mutant gene frequencies were estimated and white spotting was classified. The comparison with several other french populations supports the hypothesis that the mutant genes $O$ and $t^{b}$ have different fitnesses in country and town.

\section{Références bibliographiques}

CLARK J.M., 1975. The effects of selection and human preferences on coat color gene frequencies in urban cats. Heredity, 35, 195-210.

Committee on Standardized Genetic Nomenclature for Cats, 1968. Standardized genetic nomenclature for the domestic cat. J. Hered., 59, 39-40.

Dreux Ph., 1967. Gene frequencies in the cat population of Paris. J. Hered., 58, 89-92.

Dreux Ph., 1968. Gene frequencies in the cat population of a french rural district. J. Hered., 59, 37-39.

Dreux Ph., 1975. Génétique de population des chats domestiques de Marseille (Bouchesdu-Rhône, France). Ann. Génét. Sél. anim., 7, 23-33.

Dreux Ph., 1979. Fréquences de gènes à effets visibles dans la population des chats de la ville de Tours (Indre-et-Loire, France). Ann. Génét. Sél. anim., 11, 391-396.

Robinson R., 1959. Genetics of the domestic cat. Bibliogr. Genet., 8, 273-362.

SeARle A.G., 1968. Comparative genetics of coat colour in Mammals. Logos Press/Academic Press, New York and London, 308 p.

Todd N.B., 1969. Cat gene frequencies in Chicago and other populations of the United States. J. Hered., 60, 273-277.

Todd N.B., 1977. Cats and commerce. Sci. Am., 237 (5), 100-107.

Todd N.B., Clark J.M., Dreux Ph., 1974. Preliminary report on cat gene frequencies in northwest Scotland, Orkney and Shetland. Carn. Genet. Newsl., 2, 225-229.

Todd N.B., FAGEN R.M., FAGEN K., 1975. Gene frequencies in Icelandic cats. Heredity, 35, 172-183. 